\title{
CALR and CD47: An Insight into their Roles in the Disease Progression of MDS and MPN
}

\section{Boasman K, Simmonds MJ and Rinaldi CR}

School of Life Sciences, College of Science, University of Lincoln, Lincoln, UK

"Corresponding author: Ciro Rinaldi, School of Life Sciences, College of Science, University of Lincoln, Lincoln, UK, Tel: +44 1205 446311; E-mail: crinaldi@lincoln.ac.uk

Received date: September 12, 2018; Accepted date: November 14, 2018; Publication date: November 20, 2018

Copyright: @2018 Boasman K, et al. This is an open-access article distributed under the terms of the Creative Commons Attribution License, which permits unrestricted use, distribution, and reproduction in any medium, provided the original author and source are credited.

\begin{abstract}
Myelodysplastic syndrome and myeloproliferative neoplasms are clonal myeloid disorders arising from haematopoietic stem cells that have the tendency to progress into acute myeloid leukaemia. Multiple prognostic scoring systems have been proposed and utilised in clinical practice to predict disease evolution, however none of them can predict treatment response. In solid tumours, the relationship between the pro-phagocytic calreticulin and the anti-phagocytic $C D 47$ is repeatedly investigated. Overexpression of calreticulin has been documented to produce a pro-phagocytic signal in solid tumour and it is often counteracted by a concomitant expression of the antiphagocytic CD47 as they act in response to one another, reflecting an apoptosis vs survival mechanism in response to chemotherapy. The role of both calreticulin and CD47 are currently poorly understood in myeloid malignancies including myelodysplastic syndrome and myeloproliferative neoplasms. The aim of this review is to elaborate on the current understanding round the roles and implications of calreticulin and CD47 signalling with in solid and haematological cancers, discuss potential roles for calreticulin and CD47 expression in transformation of myeloid cells in patients with MDS or MPN into AML and how these advances are starting to be used to design new therapeutic strategies to determine disease progression and treatment response in both solid cancer and myeloid malignancies.
\end{abstract}

Keywords: Haematological cancers; Solid cancers; Myelodysplastic syndrome; Myeloproliferative neoplasms; Acute myeloid leukaemia

Abbreviations: MDS: Myelodysplastic Syndrome; MPN: Myeloproliferative Neoplasms; CALR: Calreticulin; ET: Essential Thrombocythemia; PV: Polycythaemia Vera; MF: Myelofibrosis

\section{Introduction}

Myelodysplastic syndromes (MDS), Myeloproliferative neoplasms (MPN) and acute myeloid leukaemia (AML) are myeloid malignancies which are a group of bone marrow diseases arising from clonal disorders of haematopoietic stem cells. MDS and MPN show tendencies to develop into AML as these diseases progress [1]. These diseases originate from a range of genetic events in the hematopoietic stem cells that lead to dysmyelopoiesis, abnormal differentiation, increased apoptosis with ineffective haematopoiesis, cytopenias or leucocytosis [2,3]. Scoring systems for prognosis of the disease are mainly based on bone marrow/peripheral blood features, cytogenetics and some clinical manifestations, with no specific genetic marker highlighting subtypes or stages of these conditions or predicting transformation and disease progression. In solid tumours, characterisation of the relationship between the pro-phagocytic calreticulin $(C A L R)$ and the anti-phagocytic CD47 markers is starting to show they act in response to one another, reflecting a possible apoptosis $v s$ survival mechanism in response to chemotherapy. It has been suggested that calreticulin and $C D 47$ could also play a similar role within myeloid malignancies, however their role within these diseases has been less well established. In this review using leading published data (searched for on Pubmed and Google scholar) we will discuss the current challenges in disease prognosis scoring and response to treatment within MDS and MPN, describe the current advances made in characterising the role of $C A L R$ and $C D 47$ in solid tumours and the emerging evidence for a role of $C A L R$ and $C D 47$ with in MDS and MPN. We will also evaluate how these advances in our understanding of the role of $C A L R$ and $C D 47$ are starting to aid as diagnostic markers and in therapeutic treatments for these myeloid malignancies.

\section{Challenges with Characterization and Prognostic Scoring within Myelodysplastic Syndrome and Myeloproliferative Neoplasms}

Whilst both MDS and MPN are haematopoietic stem cell disorders that tend to develop on to AML; there are distinct differences between these disease subtypes. MDS develops from a group of clonal haematopoietic stem cell disorders, which are characterised by ineffective haematopoiesis which can develop into cytopenias [4]. Prognostic scoring of MDS is usually characterized on the basis of the extent of cytopenia, the percentage infiltration of the bone marrow blasts, and abnormal chromosomal structure upon karyotyping [5]. MPNs are chronic myeloid cancers that are characterized by over production of mature blood cells, which have the potential to evolve into AML [6]. The three most common forms of MPN are Essential thrombocythemia (ET), polycythaemia vera (PV) and myelofibrosis (MF) [7], which are generally well defined from each other but quite often with overlapping presentations. Somatic mutations are common in MPNs; in the majority of patients $J A K 2$ mutations, which affect JAK/STAT pathway signalling, have been described [8]. Although other mutations in MPN have been acknowledged, such as the CALR mutations or cMPL mutations [9], it is thought that all roads lead to 
JAK/STAT pathway, with these other mutations also affecting downstream parts of the JAK/STAT pathway.

Prognosis of MDS and MPN heavily depends on type and severity of disease. Over the past 25 years classification systems (FrenchAmerican-British [FAB] and World Health Organization [WHO]) along with several prognostic-scoring systems, such as the International Prognostic Scoring System (IPSS), have been widely used $[7,10,11]$. Vast heterogeneity in these disorders results in a wide range of clinical indicators including: signs and symptoms of anaemia, infection and bleeding complications predominate, with systemic symptoms or features of autoimmunity being seen in some patients; perhaps indicative of disease pathogenesis [4].

In MDS, a standard measuring system ranking the risk factors of each disease into: very low, low, intermediate, high and very high risk is currently used (IPSS-R) [11] while in MPN a similar prognostic index (Dynamic International Prognostic Scoring System [DIPSS]) is only informative in the MF subtype, which is used for estimating survival from any point in the disease progression [12]. These scoring systems are mainly based on bone marrow/peripheral blood features, cytogenetics and some clinical manifestations such as systemic symptoms or splenomegaly but no specific genetic marker highlights specific subtypes or stages of those conditions or predicts transformation and disease progression.

Recent evidence has suggested that certain markers involved in controlling phagocytosis could be key in both disease progression and response to treatment within both solid cancer and myeloid malignancies, with the role of the pro-phagocytic $C A L R$ and antiphagocytic $C D 47$ recently coming to prominence. Overexpression of $C A L R$ has been documented to produce a pro-phagocytic signal in solid tumours [13] and it is often counteracted by a concomitant expression of the anti-phagocytic $C D 47$ [14], reflecting an apoptosis $v s$ survival mechanism in response to chemotherapy. This expression pattern could be used as a potential target for therapeutics and prognostic scoring, as $C A L R$ and $C D 47$ s role in cancers, with a potential role for $C A L R$ and $C D 47$ also emerging in MDS/MPN which will be discussed further.

\section{The Roles of Calreticulin and CD47 in Ordaining/ Controlling Phagocytosis}

CALR is an endoplasmic reticulum (ER) protein, which has a crystal structure comprise of an extended proline-rich P-domain inserted between $\mathrm{N}$ - and C-terminal domains. CALR is involved in many different functions such as calcium homeostasis, protein folding and post translational modifications [15]. Although CALR is an ER protein, it is also found throughout the cell and outside the ER is predominantly located in the membrane and the cytosol [14] where it is involved in gene expression, cell death, programmed cell removal and wound healing [16].

The structure of CALR is split into 3 different domains each with a specific function. The acidic C-domain is responsible for calcium regulation and binding, predominantly within the ER, and the globular $\mathrm{N}$-domain and extended $\mathrm{P}$-domain are primarily responsible for the protein's chaperone function $[14,15]$. The C-domain contains the KDEL amino acid sequence which prevents CALR from being secreted from the ER. Whilst the KDEL signal resides it to the ER it can however be translocated to other parts of the cell when needed [17]. KDEL-containing proteins bind to KDEL receptors on the ER affecting its translocation. If the protein is missing or has a mutated KDEL region cellular mis-localisation can occur. Localisation studies show that in myeloid cells CALR is upregulated, mutated, CALR stays located to the ER [17].

Expression of CD47 affects cellular proliferation, apoptosis, adhesion, migration and immune system homeostasis [18]. CD47 is comprised of an extracellular immunoglobulin ( $\mathrm{Ig}$ ) variable domain, a five times transmembrane-spanning domain, and a short alternately spliced cytoplasmic tail [18]. Different $C D 47$ tissue expression patterns can be seen, which has been proposed to be due to different isoforms of $C D 47$ determined by alterations in the range of length of the cytoplasmic tail from between 4-36 amino acid long [19]

The role of $C D 47$ is in vacillating and increasing $\operatorname{IgG}$ mediated phagocytosis when responding to the presence of the arginine-glycineaspartate (RGD)-containing extracellular matrix proteins [18]. As a cell surface glycoprotein of the Ig superfamily, CD47 is a transmembrane protein that interacts with several molecules including the protein signal regulatory protein alpha (SIRPa) [19,20]. CD47 blocks phagocytosis mediated by the SIRPa protein. Binding to SIRPa, CD47 stimulates the SIRPa to initiate a signalling cascade. Signalling causes recruitment of the src homology-2 domain containing protein tyrosine phosphatases SHP-1 and SHP-2, resulting in signalling to inhibit phagocytosis [21], giving CD47 the title of the "don't eat me signal" [22].

\section{$C A L R$ and $C D 47$ in Cancer Prognosis}

$C A L R$ expression on the cell surface acts as the pro-phagocytic signal activating the clearance of aged or damaged cells [23], but also, as described in different human cancers (ie AML, acute lymphocytic leukaemia, chronic myeloid leukaemia, ovarian cancer, glioblastoma, and bladder cancer [13] has roles in calcium signalling, macrophagemediated immune evasion and activation of the unfolded protein response (UPR), which is responsible for upregulation of molecular chaperones and degrading misfolded proteins. In solid tumours this is often counterbalanced by the concomitant expression of the antiphagocytic $C D 47$ [14]. Overexpression of $C A L R$ mRNA and cell surface over-exposure of $C A L R$ correlates with poor prognosis in mantle cell lymphoma, superficial and invasive bladder cancer, and neuroblastoma [13]. Chao et al. [13], showed a correlation between $C A L R$ expression and survival with high expression of CALR associating with worse survival and high counteracting over expression of $C D 47$.

In some MPN cases, mutation within exon 9 of $C A L R$ has been found, causing loss of the C-terminal acidic domain, multiple calciumbinding sites, and the KDEL sequence [24] resulting in a dysfunctional protein and contributing to over-activation of JAK/STAT pathway leading to myeloproliferation [17]. However, mutated CALR has also been shown to cause abnormal localising expressions in the cytosol and membrane [24,25] and activation of the UPR, which is often linked to disease progression via arresting protein translation through the ER compartment [26].

Several studies have shown that targeting CALR translocation to the cell membrane may represent a new strategy in cancer therapy $[27,28]$. Translocation from within the cell to the cell membrane is required for the initiation of the pro-apoptotic signalling of CALR [15]. Treatment with chemotherapy drugs anthracyclines and the reticulum $\mathrm{Ca}^{2+}$ ATPase Inhibitor thapsigargin, induces CALR translocation to the cell surface via Endoplasmic Reticulum Resident Protein 57 (ERp57) (a member of disulfide isomerase family), enhancing the immune 
response and resulting in apoptosis of the cancer cell $[27,29]$. In contrast, knocking out causes a decreased surface expression of $C A L R$ and prevents the cells from being attacked by T-cells resulting in a cell survival benefit [30].

Expression changes in $C D 47$ are a marker of phagocytosis, expression in aged or damaged cells lead to homeostatic phagocytosis. Patients suffering with cancer show high expression rates of $C D 47$ which correlate clinical outcomes. This is shown in both solid tumours [21] and also in haematological cancers including AML [31], when CD47 expression increases on the surface of cancer cells it signals for survival by evading immune surveillance, acting as a marker of antiphagocytosis $[32,33]$.

\section{Emerging Role for $C A L R$ and $C D 47$ in Myeloid Disorders}

The $C A L R / C D 47$ expression ratio seems to direct the immune response in MDS/MPN in different ways depending on which signal is predominant, with high $C A L R$ expression signalling for phagocytosis and high $C D 47$ expression protecting the cancer cells from attack by the immune system [34]. Macrophage-mediated immunosurveillance is a form of programmed cell removal (PrCR) by the immune system, where target cells are recognized and phagocytosed [35], which is independent of apoptotic removal of cells by the immune system. Studies have shown $C D 47$ to be the protector of cancer cells from the PrCR by overpowering the PrCR signals [34]. Blocking SIRPa and CD47 allows for PrCR of cancer cells and not normal cells to be targeted due to the higher expression of the eat-me signals, such as $C A L R$, which are commonly expressed in cancers in cell lines, animal models and patients [34]. The binding of CALR on the surface of a cell to LDL receptor related protein 1 (LRP1) receptor on macrophages is a vital mechanism to induce phagocytosis in targeted cells [13]. Interestingly, upregulation of CALR on the surface of cancer cells is usually followed by a concomitant upregulation in cell surface expression of the anti-phagocytic $C D 47$, in a survival response to keep the cell alive and evade immunosurveillance [34]. Cells being able to evade immunosurveillance is a key hallmark for cancer [36].

Clearance of the tumour cells can also be triggered by blocking interaction between SIRP $\alpha$ and $C D 47$, allowing for CALR phagocytosis signalling on the cell surface to become predominant [37]. MPN exhibits mutations in CALR (altering its function), seen in $67 \%$ of patients with ET and $88 \%$ of those with MF who do not have a JAK2 or MPL mutation [38]. It has been shown that only mutated CALR cells resulted in phosphorylation of STAT5 which subsequently activates the JAK/SAT pathway [39], indicating CALR to be directly involved into MPN pathogenesis and cells proliferation [40].

Role of CD47 expression in MPN and MDS has not yet been well defined and characterized, however, membrane over-expression of $C D 47$ has been measured in AML [31,41] suggesting CD47 as a potential target in AML therapy. It is known that patients with MDS, can progress on to AML throughout the course of their treatment. A gradual increase in $C D 47$ expression has been demonstrated in MDS cells progressing to AML with lower risk patients expressing the lowest level comparing with higher levels in high risk MDS. Jiang et al. [41] showed that patients with high risk MDS overexpressed $C D 47$, but exhibited lower levels of $C A L R$, while in low risk patients it was the opposite suggesting a potential role $C D 47 / C A L R$ ratio in disease progression and prognosis [13]. Interestingly, in post MDS-AML patients the level of expression of $C A L R$ is significantly lower than in de novo AML (MDS-AML: 10\%; de novo AML: 25\%) suggesting a different immune response in secondary AML and potentially a clonal evolution dictated by immune selection. Loss of $C A L R$ is detrimental to cell phagocytosis, allowing the anti-phagocytic signal $C D 47$ to evade phagocytosis resulting in a poor prognosis [13]. The role of $C A L R$ and $C D 47$ in MPN is less clear. Post MPN AML cell lines and patients do not seem to overexpress $C D 47$ as post MDS AML cell lines do suggesting a possible different mechanism of immunoresistence, however preliminary data of our laboratory, suggest an upregulation of CD47 but not CALR in chronic phase MPN (unpublished observation).

\section{$C A L R$ and $C D 47$ targeting for Anti-cancer and Myeloid Malignancy Therapy}

$C A L R$ can be used as a potential target for anti-cancer therapy using molecules able to enhance $C A L R$ expression and stimulate phagocytosis of the affected cells [15]. In prostate and lung cancer cells, $C A L R$ targeting adenovirus (Ad-CALR/MAGE-A3) have produced an up regulation of $C A L R$ and consequently an increased rate of apoptosis in the cancer cells [42]. Administration of a chimeric construct of CALR along with a chemotherapy agent increased treatment response in mice with mammary tumours [43], indicating the potential combined therapy in mammary tumours. In addition, after treatment with anthracycline, CALR expression on the cell surface correlated with the overall removal of cancer cells and survival of the patients [44], resulting in the activation of a tumour-targeting immune response in AML, neuroblastoma, non-small cell lung carcinoma,ovarian cancer, and colorectal carcinoma [44].

Anti-CD47 antibodies can block CD47-SIRPa signalling and induce cancer cells death. Dheilly et al. [45] showed how combining an antiCD49 with an anti-CD47 was effective in inducting cell death in leukemic, lymphoma, pancreatic, and ovarian carcinomas cell lines. Chemotherapy drugs are considered as the first line treatment for cancers, however, chemotherapy is not always effective. Combination therapies have been proposed, using specific chemotherapy drugs in conjunction with anti-CD47 [46]. Liu et al. [47] generated pre-clinical assessment of a humanised anti-CD47 antibody (Hu5F9-G4) which in combination with rituximab chemotherapy drug treatment, resulted in an initial reduction of all blasts in vivo. After day 159 blood counts (white blood cells, hemoglobin, and platelets), were seen to return to normal range [48]. Concomitantly, Wang et al. showed that combining cytarabine in conjunction with anti-CD47 antibody, increases the antitumor effects in AML comparing with cytarabine alone [48].

Blocking CD47 using CD47 siRNA could also represent a therapeutic strategy. Wang et al. [49] showed efficacy of CD47 siRNAs in melanoma and lung metastases regression. The CD47 siRNA were intravenously administered and resulted in reduction of tumour size. Gholamin et al. [50] demonstrated inhibition of CD47 using humanized anti- CD47 antibody Hu5F9-G4, which increases the presence of activated macrophages in brain tumours. SIRPa dependent phagocytosis induced this by blocking the CD47 signal [31]. Macrophages then become active due to the increase in the phagocytic signals, activating macrophages to phagocytose cancer cells [34]. Further studies are being carried out into both $C A K R$ and $C D 47$ targeting which makes the future of the area very interesting. 


\section{Conclusion}

The role of $C A L R$ and $C D 47$ in myeloid neoplasms is currently poorly understood. In solid tumours overexpression of $C A L R$ produces a pro-phagocytic signal and it is often counteracted by a concomitant expression of the anti-phagocytic CD47. Over expression of $C A L R$ has been seen to correlate with poor prognosis as it is counteracted by increased $C D 47$ increased expression, which is critical for cancer cell survival. Several studies demonstrated how CD47 is often overexpressed in AML, allowing the leukaemia cell to evade immune surveillance and survive. During MDS progression onto AML, the levels of expression of CALR increase but is immediately counteracted by the concomitant $C D 47$ overexpression, resulting in a predominate anti-phagocytic message. The expression of $C D 47$ in MPN is not well defined yet. Preliminarily data from our laboratory indicate in MPN samples no increase of $C A L R$ expression is seen on the cell surface, whilst $C D 47$ expression is significantly elevated in ET, PV and MF, indicating a possible different mechanism of immunoresistance comparing to solid tumour and AML.

Emerging therapeutic strategies targeting $C A L R$ and $C D 47$ expression have offered an interesting insight into the roles of $C A L R$ and $C D 47$ in myeloid neoplasms and their implication into pathogenesis and disease progression. Targeting $C A L R$ with the aim of increasing its expression on the cell surface using an adenovirus (AdCALR/MAGE-A3) resulted in significant increase in cell death and apoptosis. Blocking the CD47-SIRPa signalling pathway by using Hu5F9-G4 has resulted in reduction of $C D 47$ on cancers cells and consequently increases in cancer cell removal and tumour burden reduction.

$C A L R$ and $C D 47$ play vital roles in the pro/anti - phagocytic mechanisms of cancer cells. Better understanding is required for their involvement in myeloid malignancies. CALR and CD47 targeted therapies alone or in combination with standard chemotherapy could result in better outcome for patients suffering with both haematological and solid cancers. Further studies are required to better fully understand the implication of CALR and CD47 expression/ regulation and their prognostic role in disease progression and treatment response in cancer and the myeloid malignancies.

\section{References}

1. Korn C, Méndez-Ferrer S (2017) Myeloid malignancies and the microenvironment. Blood 129: 811-822.

2. Larsson CA, Cote G, Quintás-Cardama A (2013) The changing mutational landscape of acute myeloid leukemia and myelodysplastic syndrome. Mol Cancer Res 11: 815-827.

3. Yang X, Yao R, Wang H. Update of ALDH as a potential biomarker and therapeutic target for AML. Biomed Res Int 2018: 1-5.

4. Adès L, Itzykson R, Fenaux P (2014) Myelodysplastic syndromes.The Lancet 383: 2239-2252.

5. Gill H, Leung A, Kwong Y-L (2016) Molecular and cellular mechanisms of myelodysplastic syndrome: Implications on targeted therapy. Int J Mol Sci 17: 440 .

6. Park JH, Sevin M, Ramla S, Truffot A, Verrier T, et al. (2015) Calreticulin mutations in myeloproliferative neoplasms: Comparison of three diagnostic methods. PLoS One 10 E0141010.

7. Vardiman JW, Thiele J, Arber DA, Brunning RD, Borowitz MJ, et al (2009) The 2008 revision of the World Health Organization (WHO) classification of myeloid neoplasms and acute leukemia: Rationale and important changes. Blood 114: 937-951.

8. Campbell PJ, Baxter EJ, Beer PA, Scott LM, Bench AJ, et al. (2006) Mutation of JAK2 in the myeloproliferative disorders: Timing, clonality studies, cytogenetic associations, and role in leukemic transformation. Blood 108: 3548-3555.

9. Tefferi A, Pardanani A (2014) CALR mutations and a new diagnostic algorithm for MPN. Nat Rev Clin Oncol 11: 125-126.

10. Jonas BA, Greenberg PL (2015) MDS prognostic scoring systems - past, present, and future. Best Pract Res Clin Haematol 28: 3-13.

11. Vardiman J (2012) The classification of MDS: From FAB to WHO and beyond. Leukemia Research 36: 1453-1458.

12. Gangat N, Caramazza D, Vaidya R, George G, Begna K, et al. (2011) DIPSS plus: A refined dynamic international prognostic scoring System for primary myelofibrosis that incorporates prognostic information from karyotype, platelet count, and transfusion status. J Clin Oncol 29: 392-397.

13. Chao MP, Jaiswal S, Weissman-Tsukamoto R, Alizadeh AA, Gentles AJ, et al. (2010) Calreticulin is the dominant pro-phagocytic signal on multiple human cancers and is counterbalanced by CD47. Sci Transl Med 2: 63 ra94.

14. Chao MP, Gotlib J (2014) Two faces of ET: CALR and JAK2. Blood 123 1438-1440.

15. Michalak M, Groenendyk J, Szabo E, Gold LI, Opas M (2009) Calreticulin, a multi-process calcium-buffering chaperone of the endoplasmic reticulum. Biochem J 417: 651-666.

16. Araki M, Komatsu N (2017) Novel molecular mechanism of cellular transformation by a mutant molecular chaperone in myeloproliferative neoplasms. Cancer Sci 2017 108: 1907-1912.

17. Guglielmelli P, Nangalia J, Green AR, Vannucchi AM (2014) CALR mutations in myeloproliferative neoplasms: Hidden behind the reticulum. AJH Educational Material 89: 453-456.

18. Oldenborg P-A, Per-Arne (2013) CD47: A cell surface glycoprotein which regulates multiple functions of hematopoietic cells in health and disease. ISRN Hematol 2013: 1-9.

19. Jaiswal S, Jamieson CHM, Pang WW, Park CY, Chao MP, et al. (2009) CD47 is upregulated on circulating hematopoietic stem cells and leukemia cells to avoid phagocytosis. Cell. 2009 138: 271-285.

20. Golubovskaya V, Berahovich R, Zhou H, Xu S, Harto H, et al. (2017) CD47-CAR-T cells effectively kill target cancer cells and block pancreatic tumor growth. Cancers Basel 9: 139.

21. Willingham SB, Volkmer JP, Gentles AJ, Sahoo D, Dalerba P, et al. (2012) The CD47-signal regulatory protein alpha (SIRPa) interaction is a therapeutic target for human solid tumors. Proc Natl Acad Sci USA 109: 6662-6667.

22. Brightwell RM, Grzankowski KS, Lele S, Eng K, Arshad M, et al. (2016) The CD47 \&quot;don't eat me signal\&quot; is highly expressed in human ovarian cancer. Gynecol Oncol 143: 393-397.

23. Gardai SJ, McPhillips KA, Frasch SC, Janssen WJ, Starefeldt A, et al. (2005) Cell-surface calreticulin initiates clearance of viable or apoptotic cells through trans-activation of LRP on the phagocyte. Cell 123: 321-334.

24. Nangalia J, Massie CEE, Baxter EJJ, Nice FLL, Gundem G, et al. (2013) Somatic CALR mutations in myeloproliferative neoplasms with nonmutated JAK2. N Engl J Med 369: 2391-2405.

25. Klampfl T, Gisslinger H, Harutyunyan AS, Nivarthi H, Rumi E, Milosevic JD, et al. (2013) somatic mutations of calreticulin in myeloproliferative neoplasms. N Engl J Med 369: 2379-2390.

26. Ma Y, Hendershot LM (2004) The role of the unfolded protein response in tumour development: Friend or foe? Nat Rev Cancer 4: 966-977.

27. Obeid M, Tesniere A, Panaretakis T, Tufi R, Joza N, et al. (2007) Ectocalreticulin in immunogenic chemotherapy.Immunological Reviews 220 : 22-34.

28. Tesniere A, Panaretakis T, Kepp O, Apetoh L, Ghiringhelli F, et al. (2008) Molecular characteristics of immunogenic cancer cell death. Cell death differ 15: 3-12.

29. Jeffery E, Peters LR, Raghavan M (2011) The polypeptide binding conformation of calreticulin facilitates its cell surface expression under conditions of ER stress. J Bio chem 286: 2402-2415. 
Citation: Boasman K, Simmonds MJ, Rinaldi CR (2018) CALR and CD47: An Insight into their Roles in the Disease Progression of MDS and MPN. J Blood Disord Transfus 10: 1000413. doi:10.4172/2155-9864.1000413

Page 5 of 5

30. Xu Q, Chen C, Lin A, Xie Y (2017) Endoplasmic reticulum stressmediated membrane expression of CRT/ERp57 induces immunogenic apoptosis in drug-resistant endometrial cancer cells. Oncotarget 8: 58754-58764.

31. Majeti R, Chao MP, Alizadeh AA, Pang WW, Jaiswal S, et al. (2009) CD47 is an adverse prognostic factor and therapeutic antibody target on human acute myeloid leukemia stem cells. Cell 138: 286-299.

32. Galli S, Zlobec I, Schürch C, Perren A, Ochsenbein AF, et al. (2015) CD47 protein expression in acute myeloid leukemia: A tissue microarray-based analysis. Leuk Res 39: 749-756.

33. Tsai RK, Rodriguez PL, Discher DE (2010) Self inhibition of phagocytosis: The affinity of "marker of self" CD47 for SIRPalpha dictates potency of inhibition but only at low expression levels. Blood Cells Mol Dis 45: 67-74.

34. Feng M, Chen JY, Weissman-Tsukamoto R, Volkmer J-P, Ho PY, et al. (2015) Macrophages eat cancer cells using their own calreticulin as a guide: Roles of TLR and Btk. Proc Natl Acad Sci U S A 112: 2145-2150.

35. Chao MP, Majeti R, Weissman IL (2012) Programmed cell removal: A new obstacle in the road to developing cancer. Nat Rev Cancer 12: 58-67.

36. Hanahan D, Weinberg RA (2011) Hallmarks of Cancer: The Next Generation. Cell 144: 646-674.

37. Reya T, Morrison SJ, Clarke MF, Weissman IL (2001) Stem cells, cancer and cancer stem cells. Nature 414: 105-111.

38. Saeidi K (2016) Myeloproliferative neoplasms: Current molecular biology and genetics. Crit Rev Oncol Hematol 98: 375-389.

39. Lavi N (2014) Calreticulin mutations in myeloproliferative neoplasms Rambam Maimonides Med J 5: e0035.

40. Yi L, Shan J, Chen X, Li G, Li L, et al. (2016) Involvement of calreticulin in cell proliferation, invasion and differentiation in diallyl disulfide-treated HL-60 cells. Oncol Lett 12: 1861-1867.

41. Jiang H, Fu R, Wang H, Li L, Liu H, et al.(2013) CD47 is expressed abnormally on hematopoietic cells in myelodysplastic syndrome. Leuk Res 2013 37: 907-910
42. Liu X, Sun N, Dong Y, Li J, Liu Y, et al. (2015) Anticancer effects of adenovirus-mediated calreticulin and melanoma-associated antigen 3 expression on non-small cell lung cancer cells. Int Immunopharmacol 25: 416-424.

43. Obeid M, Tesniere A, Ghiringhelli F, Fimia GM, Apetoh L, et al. (2007) Calreticulin exposure dictates the immunogenicity of cancer cell death. Nat Med 13: 54-61.

44. Fucikova J, Truxova I, Hensler M, Becht E, Kasikova L, et al. (2016) Calreticulin exposure by malignant blasts correlates with robust anticancer immunity and improved clinical outcome in AML patients. Blood 128: 3113-3124.

45. Dheilly E, Moine V, Broyer L, Salgado-Pires S, Johnson Z, et al. (2017) Selective blockade of the ubiquitous checkpoint receptor cd 47 is enabled by dual-targeting bispecific antibodies. Mol Ther 25: 523-533.

46. Xiang YR, Liu L (2017) "Eating" Cancer cells by blocking CD47 signaling: Cancer therapy by targeting the innate immune checkpoint. Cancer Transl Med 3: 200.

47. Liu J, Wang L, Zhao F, Tseng S, Narayanan C, et al. (2015) Pre-clinical development of a humanized anti-cd47 antibody with anti-cancer therapeutic potential. PLoS One 10: e0137345.

48. Wang Y, Yin C, Feng L, Wang C, Sheng G, et al. (2015) Ara-C and antiCD47 antibody combination therapy eliminates acute monocytic leukemia THP-1 cells in vivo and in vitro. Genet Mol Res 14: 5630-5641.

49. Wang Y, Xu Z, Guo S, Zhang L, Sharma A, et al. (2013) Intravenous delivery of sirna targeting cd47 effectively inhibits melanoma tumor growth and lung metastasis. Mol Ther 21: 1919-1929.

50. Gholamin S, Mitra SS, Feroze AH, Liu J, Kahn SA, et al. (2017) Disrupting the CD47-SIRPa anti-phagocytic axis by a humanized antiCD47 antibody is an efficacious treatment for malignant pediatric brain tumors. Sci Transl Med 9:eaaf2968. 